TTR

Traduction, terminologie, re?daction

\title{
Soumission et assujettissement : la fidélité chez les traducteurs et « théoriciens " de la traduction française dans la première moitié du XVIII ${ }^{\mathrm{e}}$ siècle
}

\section{Benoit Léger}

Volume 9, numéro 2, 2e semestre 1996

Parcours de traduction

Pathways of Translation

URI : https://id.erudit.org/iderudit/037259ar

DOI : https://doi.org/10.7202/037259ar

Aller au sommaire du numéro

Éditeur(s)

Association canadienne de traductologie

ISSN

0835-8443 (imprimé)

1708-2188 (numérique)

Découvrir la revue

Citer cet article

Léger, B. (1996). Soumission et assujettissement : la fidélité chez les traducteurs et « théoriciens " de la traduction française dans la première moitié du XVIII ${ }^{\mathrm{e}}$ siècle. TTR, 9(2), 75-101. https://doi.org/10.7202/037259ar
Résumé de l'article

Soumission et assujettissement : la fidélité chez les traducteurs et " théoriciens " de la traduction française dans la première moitié du XVIIIe siècle - Cet article porte sur le concept de fidélité en traduction en France au XVIIIe siècle. À partir des définitions des dictionnaires de la fin du XVIIe et du début du XVIIIe siècle, on cherche à définir la visée de la fidélité à l'époque : fidélité au texte de départ ou à la culture d'arrivée? Les dictionnaires définissent d'abord le terme par rapport à l'exactitude, à la foi et à la soumission. Les débats sur la fidélité dans la traduction des Anciens entre Houdar de La Motte et Anne Dacier au début du XVIIIe siècle se reflètent dans la traduction de la littérature anglaise. L'assujettissement du traducteur devient avec Silhouette celui du copiste. La fidélité est associée par Desfontaines à l'exactitude, mais en tant qu'idéal elle ne peut être selon lui que soumise aux contingences de la traduction et de la langue française. La fidélité devient alors synonyme de littéralité et Desfontaines, comme d'autres traducteurs, choisit plutôt d'être fidèle au goût français. L'exigence même de fidélité se module selon le type de texte à traduire : la Bible exigera une version littérale, les Anciens une imitation cherchant même à rendre justice à l'auteur en l'« améliorant ", et les auteurs contemporains dont la " gloire " n'est pas assez importante, n'auront droit qu'à une adaptation libre.
Tous droits réservés @ C TTR: traduction, terminologie, rédaction — Les auteurs, 1996
Ce document est protégé par la loi sur le droit d'auteur. L'utilisation des services d'Érudit (y compris la reproduction) est assujettie à sa politique d'utilisation que vous pouvez consulter en ligne. 


\section{Soumission et assujettissement : la fidélité chez les traducteurs et " théoriciens » de la traduction française dans la première moitié du XVIII ${ }^{\mathbf{e}}$ siècle $^{1}$}

\section{Benoit Léger}

\section{Introduction}

Encore plus que cẹlle de l'équivalence ou de l'imitation, la question de la fidélité fait partie de presque tous les débats sur le rôle et les règles de la traduction au XVII ${ }^{e}$ siècle. Les écrits de l'époque comportent de nombreuses références à la fidélité et à ses dérivés

1. Cet article présente certains aspects d'une analyse du vocabulaire de la traduction dans la première partie du XVII' sižcle français qui s'intégrera à ma thèse de doctorat sur la poetique traductionnelle de Desfontaines. Les recherches qui ont mene à ja rédaction de cet article ont été rendues possibles grace à une bourse de doctorat du Conseil de recherche en sciences humaines du Canada (CRSHC) et à une bourse pour séjour à l'étranger du Fonds pour la formation de chercheurs et l'aide à la recherche (FCAR). Je tiens à remercier ici Mesdames Chantal Bouchard et Annick Chapdelaine, professeures au Département de langue et litterature françaises (McGili), ainsi que Jennifer Looper et Andrea Petruschke pour le temps qu'elles ont consacré a lire cet article et à me faire part de leurs suggestions. 
(fidelle, infidelle, [in]fidellement) ${ }^{2}$. Cet article s'inscrit donc dans une tentative de description "archéologique ${ }^{3}$ " afin de mieux comprendre le vocabulaire du discours sur la traduction littéraire en France, des débuts de la Régence en 1715 jusqu'aux années 1740. Cela devrait contribuer à définir les discours sur la traduction qui cohabitent et ainsi éventuellement à établir qu'il existe une poétique traductionnelle prédominante opposée à des discours coexistants et divergents.

L'étape préliminaire de cette recherche a été constituée par une première lecture de textes d'époque, dans le but de repérer puis de définir certaines des composantes du vocabulaire dont disposent les traducteurs ainsi que les critiques et " théoriciens " de cette même période. L'importance du rổe joué par le vocabulaire a bien sûr été soulignée par Foucault (1969, pp. 65-66), pour qui l'analyse des contenus lexicaux définit les éléments de signification dont disposent les sujets parlants à une époque donnée, ainsi que la structure sémantique qui apparaît à la surface des discours déjà prononcés. Nous nous sommes intéressé ici particulièrement au premier rôle de l'analyse des contenus lexicaux, soit la définition des éléments de signification. Notre démarche préalable vise donc à cerner, autant que faire se peut, les éléments contradictoires qui joueront dans le discours sur la traduction.

La lecture de Foucault par St-Pierre (1993), dans le domaine de la théorie de la traduction, a constitué le point de départ de notre démarche : St-Pierre reprend les questions de Foucault sur les règles qui gouvernent la production du discours : ce qui peut être dit (le

2. Nous avons choisi de conserver la graphie de tous les termes et extraits d'époque cités dans cet article afin d'eviter de les " traduire » inutilement, de même que pour bien souligner le décalage qui existe entre le sens que nous attribuons aujourd'hui a ces memes termes et celui qu'ils ont aux XVII* et XVIII siecles.

3. Michel Foucault (1969); sur l'« archéologie w de la traduction, cf. Antoine Berman, l'Épreuve de l'étranger (1984). 
dicible, « sayable "), la conservation, la mémoire, la réactivation et l'appropriation. La première catégorie nous intéressera ici, le « sayable » devenant ce que St-Pierre appelle les « limites et formes du traduisible ". L'étude de ces limites soulève des questions comme : qu'est-il possible de traduire à une époque donnée; quels sont les critères de traduction de différents types de discours; comment les différentes aires de discours se définissent et se délimitent-elles? Une première réponse à ces différentes questions devrait se trouver dans l'étude du paratexte et du péritexte des traducteurs et théoriciens de la traduction, et dans leur mise en rapport. On arriverait ainsi à definir les éléments de signification dont les sujets parlants disposent à l'époque considérée.

Nous avons procédé à ce recensement du vocabulaire de la traduction à partir des méthodes de travail terminologique, en recherchant particulièrement les occurrences de certains mots clés, de certains concepts reliés à la traduction littéraire, repérés au cours de notre lecture préalable des textes. Il s'est cependant rapidement avéré qu'il fallait faire appel à des textes antérieurs pour mieux comprendre le contexte dans lequel s'inscrira la poétique traductionnelle entre 1715 et 1740 : nous avons donc tenu compte d'écrits de certains théoriciens et de dictionnaires de la seconde moitiê du XVII" siècle. Le corpus recensé comporte ainsi trois types de textes : $1^{*}$ les définitions de termes et de concepts tirées d'ouvrages lexicographiques, linguistiques et généraux antérieurs ou contemporains, ou, dans certains cas, ultérieurs (les dictionnaires et, dans une moindre mesure, l'Encyclopédie et les grammaires), $2^{\circ}$ le paratexte des traducteurs (explications ou justifications dans les préfaces et les "notes du traducteur") et $3^{*}$ les critiques de journalistes et d'essayistes de l'époque. Dans le cas du paratexte des traducteurs et des écrits critiques, nous avons accordé une large place au discours de l'abbé Desfontaines dont l'activité de " polygraphe " touche tant à la traduction qu'à la critique littéraire.

Considérant la durée de vie des dictionnaires, nous avons ici utilisé des ouvrages publies d'abord à la fin du XVII siècle, dont Matoré (1968) souligne l'importance et mentionne les rééditions au cours du siècle suivant. Ce type d'ouvrage, par essence normatif et 
souvent passéiste ou puriste (Matoré, 1968, p. 25), ne saurait de façon isolée définir le vocabulaire de la traduction; en tant qu'ouvrages de référence et tentative de compilation des connaissances et des idées d'une époque, ils constituent cependant de précieux instruments de travail. Les dictionnaires utilisés sont donc le Dictionnaire français (Richelet, 1680), le Dictionnaire universel (Furetière, 1690) et le Nouveau Dictionnaire de l'Académie françoise (1718).

Si le nombre de dictionnaires publiés entre la fin du $\mathrm{XVII}^{\circ}$ siècle et le milieu du XVIII ${ }^{\circ}$ est relativement facile à délimiter, il en va tout autrement des très nombreuses traductions et donc du paratexte traductionnel. Nous avons ainsi retenu un certain nombre de prefaces de traducteurs, soit parce qu'elles semblaient représentatives de la poétique traductionnelle dominante ou officielle (Dacier, Desfontaines), soit pour leur valeur polémique (Silhouette, Houdar de La Motte). Nous avons évidemment accordé une importance particulière aux prefaces de traducteurs de romans anglais tels que Desfontaines, puisqu'elles relevaient plus directement de notre champ de travail. Mais nous avons, à cette étape, tenu compte d'autres prefaces de traductions, tant celles d'auteurs anciens (Dacier, Silhouette, Houdar de La Motte) que contemporains et en particulier anglais (Desfontaines, Silhouette, Yart), ou encore de traductions de différents types d'ouvrages (traités philosophiques, recueils poétiques, ouvrages historiques, journaux) par Desfontaines, encore une fois parce qu'elles s'inscrivent dans la poétique traductionnelle dominante, ou parce qu'elles présentent des points de vue polémiques.

Nous nous sommes ici particulièrement arrêté aux nombreuses analyses de traductions par Desfontaines dans deux des trois revues littéraires qu'il a publiées de 1731 à 1746 et qui font de lui «l'un des premiers journalistes littéraires » en France (Morris, 1961, p. 20): les Observations sur les écrits modernes (Desfontaines, 1735-1743) et les Jugements sur quelques ouvrages nouvearxx (Desfontaines, 1744-1746). 


\section{La « fldélité » à la fin du XVIr" siècle}

Revenons d'abord sur l'importance de la "fidélité » pour les grammairiens et linguistes du XVII' siècle : ainsi pour Méziriac, la seule " beauté du langage " n'est pas le seul critère de qualité d'une traduction; la fidélité en constituerait la qualité la plus importanté. Des traducteurs tels que Longepierre considèrent également que la fidélité est la qualité majeure d'une traduction (Guellouz, 1991, p. 263). Pour Godeau, le rôle principal du traducteur doit être de rendre " le sens avec une exacte fidélité ", tout en tenant compte des différences de goût :

et ce seroit quelque fois une faute de jugement trìs signalée que de s'amuser à la forme de l'élocution, chaque nation ayant des gouts très différents pour les grâces du style, et ce qui excite l'admiration en un endroit, courant fortume de n'être pas souffert en un autre.

On note que le classicisme français n'a pas encore convaincu de son universalité : Godeau reconnaît ici aux différents pays, aux différentes " nations» (on n'ose écrire " cultures ») le droit à des « goûts très différents ».

Le concept de fidélité n'en reste pas moins vague : en tant que grand principe « moral », tous semblent s'entendre sur son importance, mais on se demande pourtant encore de quelle fidélité il s'agit : à la langue, au texte, à la culture de départ, ou fidélité au polysystème linguistique, littéraire et culturel français du classicisme? A posteriori la réponse est prévisible, mais force est de

4. « La beauté du langage ne suffit pas pour faire estiner une traduction excellente. Il n'y a personne qui n'avoue que la qualite la plus essentielle à un bon traducteur, c'est la fidélité. " (Méziriac, « De la traduction ", 1635, cité par Ballard, 1992, p. 164.)

5. Godeau, " Discours sur les ceuvres de M. Malherbe ", 1630, cité par Ballard (1992), p. 159. 
constater que les premières évocations du concept et ses premières définitions ne donnent pas de réponse explicite à cette question. On trouve cependant des éléments de réponse dans la poétique traductionnelle de Perrot d'Ablancourt et de Huet.

La question de la fidélité au XVII ${ }^{\circ}$ siécle est bien sûr indissociable des écrits théoriques et des traductions de d'Ablancourt qui jouissent à l'époque d'un grand succès, ainsi que de la reconnaissance d'une partie de la république des lettres. Richelet lui rendra ainsi hommage dans l' " Avertissement " de son Dictionnaire et l'évoquera à plusieurs reprises dans ses définitions : " Traducteur. (...) [Les fameux \& les excellens Traducteurs François ce sont d'Ablancourt, Vaugelas, Messieurs de Port-Royal, d'Andilli \& quelques autres en fort petit nombre.] " Il serait trop long d'analyser ici en détail la poétique traductionnelle de d'Ablancourt pour qui le texte étranger doit se plier au gout français selon le principe des fameuses "belles infidèles »; on se contentera de renvoyer aux travaux de $\mathrm{R}$. Zuber (1968) et de rappeler la critique de Chapelain ${ }^{6}$ sur d'Ablancourt.

Cette critique de Chapelain a en effet le mérite de montrer encore une fois que des points de vue divergents s'opposent à la poétique traductionnelle dominante à la fin du XVIr siècle. Dans cette poétique dominante, la fidélité est le propre d'une traduction qui s'intègre sans heurt, fidèlement, à la culture classique de langue française $^{\gamma}$ et qui gomme toute caractéristique (culturelle ou

6. Chapelain, Lettres, T. de Larroque, dir. Paris, 1883, t. 2, p. 212; cité par Bury (1991), p. 252.

7. La traduction française permettra ensuite, au XVIII siècle, la diffusion de la litterature etrangère, en particulier anglaise, destinée au public "érudit » de l'époque qui lit le français (Réau, 1938 et 1971). Le rejet de l'étranger devra done, à un degré moindre, se refléter dans le goût des lecteurs étrangers francophones. 
formelle) étrangère. Avant de respecter la fidélité philologique, le texte traduit doit être efficace.

La poétique traductionnelle de Huet, à la même époque, vient pourtant s'opposer à celle de d'Ablancourt; selon Huet, le meilleur « modele de traduction » est celui où le traducteur

s'attache très étroitement a la pensée de l'auteur, puis aux mots même si les possibilités offertes par les deux langues le permettent, et enfin où il reproduit le style personnel de l'auteur autant que faire se peut, s'appliquant seulement à le représenter fidelement, sans le diminuer par aucune suppression nj l'augmenter d'aucune addition, mais dans son intégrité et le plus ressemblant possible en tous points."

Huet semble donc être un des premiers théoriciens de notre corpus à definir un peu plus concrètement la fidélité et ses applications : le traducteur fidèle doit suivre la pensée de l'auteur, les mots et le style et, comme le souligne Bury (1991, p. 256),

[ii] ne doit ni retrancher ni ajouter, mais il doit garder intact son modèle, comme devrait étre le portrait [...] de l'auteur, qui méme absent semble present devant nous [...]. On est ici à l'opposé de la « naturalisation " que pratique d'Ablancourt.

Cette fidélité, au lieu de demeurer un concept abstrait, s'inscrit chez Huet dans une triade : " le scrupule (religio) dans l'exposé de la pensée, la fidélité (fides) dans la reproduction des mots, et le plus grand soin (summa sollicitudo) dans l'expression de la couleur de l'auteur. " (Bury, 1991, p. 257)

Encore une fois, la poétique divergente de Huet et de Chapelain, si elle ne semble pas avoir eu l'accueil de celle de

8. Chapelain, cité par Bury (1991), p. 252.

9. Huet, De interpretatione (1661), cité et traduit par Bury (1991), p. 256. 
d'Ablancourt et des tenants de l'annexion par fidélité à la culture d'arrivée, atteint cependant le niveau nécessaireà sa diffusion et l'on peut supposer que les théoriciens, critiques et traducteurs du XVIII ${ }^{\circ}$ s'inscriront dans la tradition de l'une ou l'autre poétique.

Notons également que l'usage du terme " fiólitié " est confirmé chez les théoriciens du XVII siècle : le sens qu'ils lui attribuent differe, mais Méziriac, Chapelain, Tourreil, Huet et d'Ablancourt l'utilisent, de même que ses dérivés.

\section{Les lexicographes et la " fidélité *}

Les dictionnaires de la fin du XVII siècle et du début du XVIII ne semblent pas de prime abord offrir de conclusions plus intéressantes au sujet des concepts de fidélité et d'infidélité, mais la comparaison des définitions et des liens établis entre les différents sens, permet de nuancer les conceptions de la fidélité.

Pour Richelet d'abord, la fidélité est une " [s]orte de vertu qui consiste à observer éxactement \& sincérement ce qu'on a promis. " Le concept est ensuite associé à la loyauté et au devoir mais aucunement à la traduction. Ce qui est " infidéle " est donc par conséquent ce qui " n'a point de fidélité, qui a manqué à sa foi. " Il est curieux de retrouver ici des citations attribuees à d'Ablancourt aux entrées " fidéle " ("Le chien est fidèle à son maître "), " fidélement » ( Servir fidélement ») et « infidelle » ( Etre infidéle à son maître $»)$.

La définition de Furetière reprend l'association à la foi et à la soumission du serviteur ou du chien à leur maître, mais lui ajoute une définition relative à la vérité et à la traduction : " Fidelle, signifie aussi, Veritable. [...] Une traduction fidelle, est celle qui ne change point le sens de son Auteur, qui n'y ajouste, ni n'y diminue. " Pour Furetière toujours, ce qui est " infidelle " se dit en " morale » de ce qui " n'est pas conforme à la vérité »; il donne comme exemples : "Une copie infidelle, qui ne ressemble point à l'original. un portrait infidelle. " 
Le Nouveau Dictionnaire de l'Académie (1718) reprend l'essentiel de cette définition, en donnant cependant des exemples différents pour la traduction: la fidélité est d'abord définie par rapport à la loyauté et à la foi (" garder fidelité à son Prince "; " une femme doit fidelité à son mari "). Le deuxième sens est encore celui de l'exactitude, de la conformité à la vérité : fidélité de l'historien, d'un rapport ou d'une traduction (" cet Autheur est traduit avec fidelite ${ }^{10} \mathrm{M}$ ).

On constate que lé terme de fidélité, qui n'était pas associé à la traduction chez Richelet, le devient chez ses successeurs. Dans tous les cas, la fidélité est définie par deux types de rapports de force : la foi (étymologie) et la soumission. Il n'est pas sans intérêt de souligner que la fidélité, associée de nos jours au sentiment amoureux, ou, en traduction, à la conformité à un texte étranger, est synonyme ici de soumission à un credo ou à un pouvoir, tandis que l'association à la vérité ou à l'équivalence semble, pour les lexicographes, plus récente, "fidel(le) " signifiant " aussi " " conforme à la vérité " pour Furetière. Les conclusions à tirer de ce triangle sémantique foi-soumission-vérité semblent précaires à ce niveau de notre travail; on peut quand même se demander si le concept de fidélité ne prendra pas pour les traducteurs un sens différent selon le type de texte traduit.

\section{La « fidélité " pour les traducteurs du XVIII" siècle}

Les deux conceptions de la fidélité (à la culture et au texte étrangers, ou au polysystème classique français) continuent de coexister au XVIII" siècle dans le discours theorique des traducteurs. Avant de passer au point de vue de Desfontaines, nous avons retenu ici celui de Dacier, Houdar de La Motte, Silhouette, Fréron et Yart, de même que celui d'un traducteur anonyme cité par Graeber (1990).

10. La définition de l'adjectif reprend l'association à la loyauté et à la foi, puis à la vérité : « histoire fidelle, copie fidelle, traduction fidelle....) 
Les débats sur la notion de fidélité au début du $\mathrm{XVIII}^{\circ}$ siècle se poursuivent dans le contexte de la seconde querelle des Anciens et des Modemes et semblent porter, pour l'essentiel, sur la fidélité de la prose lorsqu'il s'agit de la traduction d'un texte en vers, ainsi que sur le degré de fidélité nécessaire. La question se pose d'abord pour la traduction des Anciens, mais également, plus tard, pour celle des auteurs contemporains (les Anglais en particulier).

La publication de la traduction de l'lliade par Houdar de La Motte en 1714 donne ainsi lieu à un véritable pamphlet de la part de l'érudite Dacier qui s'insurge contre les infidélités commises. Dacier avait déjà publié une traduction de l'hiade dans laquelle elle expliquait avoir choisi la traduction en prose parce que le traducteur peut alors dire

tout ce qu'Homère a dit; c'est ce qu'il ne peut jamais faire en vers, surtout en notre langue, où il faut nécessairement qu'il change, qu'il retranche, qu'il ajoute. Or, ce qu'Homere a penst et dit, quoique rendu plus simplement et moins poétiquement qu'il ne l'a dit, vaut certainement mieux que tout ce qu'on est forcé de lui prêter en le traduisant en vers."

De plus, le français de l'époque n'a pas pour Dacier, comme pour tant de disciples des Anciens, la capacité d'atteindre à la beauté des vers d'Homère.

Dans les dernières pages de son " Discours sur Homère ", Houdar de La Motte présente la poétique traductionnelle qui l'a guidé et en profite pour critiquer la traduction en prose de Dacier. Celle-ci riposte par son Des causes de la corruption du goût (1714) où elle analyse dans le détail la position de Houdar de La Motte et s'insurge contre toute tentative d'adaptation, de transposition ou d'imitation du texte d'Homère qui doit être religieusement et humblement traduit : si Houdar de La Motte, en bon Moderne,

11. Dacier, "Introduction ", I'lliade, Paris, 1714, citée par P.A. Horguelin (1981), p. 114. 
considère que l'on peut surpasser ce qu'Homère a écrit, Dacier, elle, se moque de cette présomption, elle qui dit avoir fait preuve d'humilité devant le texte et n'avoir pas vu les fautes qque Houdar de La Motte voulait y corriger :

moy qui, sans m'apercevoir des défauts infinis, qui sont dans Homere, l'ay traduit en Prose le plus littéralement \& le plus fidellement qu'il m'a été possible, \& qui en mille endroits ay esté assez simple pour avoler tres sincerement que je me reconnoissois tres inferieure à mon Original [...] : \& M. de la Motte qui avec un genie superieur vient nous ouvrir les yeux, \& nous faire voir les beviles innombrables de ce Pokte; \& qui non seulement s'est cru capable de le corriger, mais encore de l'embellir ! (Dacier, 1735, 1970, pp. 11-12)

Notons ici l'association prose-lettre-fidélité, la prose étant vue comme le meilleur moyen de traduire fidèlement un texte quasi sacré auquel il est interdit de toucher ${ }^{12}$. Bien sur, dans les faits, Dacier et Houdar de La Motte partagent certaines pratiques traduisantes de leur époque (dont le rejet de tout ce qui est considéré comme " bas " (Horguelin, 1981, p. 113), ce qui fait dire à Horguelin citant Cary que le résultat final des traductions de Dacier et de Houdar de La Motte est plus proche que les projets distincts qui les séparaient : « En dépit de leurs professions de foi contraires, ces deux traducteurs vivaient à la même époque et traduisaient pour le même public : et cette contrainte-là se révèle plus agissante que leurs intentions déclarées. " (Cary, 1963, p. 34) Il n'en demeure pas moins que deux théories, deux poétiques s'affrontent ici dans une surenchère de " réponses » (Houdar de La Motte répondra encore à Dacier par ses Réflexions sur la critique en 1715) : la fidélité (fides) au texte sacré d'un Ancien et la fidélité (soumission) aux Modernes.

Si la querelle de Dacier et de Houdar de La Motte concerne la traduction d'un des textes canoniques de la littérature, le traducteur anonymie du Free-Holder prend cependant parti en 1727

12. «[...] Dacier [...] se prononce pour la prose, parce qu'elle estime avant tout la fidélité " (Morris, 1961, p. 323). 
contre les transpositions en parlant d'abord de la traduction d'un texte moins canonique, celle du Spectator :

[...] je l'ai confrontée avec l'Original, souvent en compagnie de gens, qui entendent à fonds les deux Langues, \& qui m'ont fait remarquer plus d'une fois, non seulement ce tour heureux à rendre certaines choses, qui, sans doute, donneroient de l'exercice à ceux qui censurent le plus cavalierement, mais encore la fidelité de la Traduction. Car, tout le monde ne conviendra pas, avec l'Auteur que je viens de citer [...] : Pour bien traduire les Ouvrages de cette espèce, il faut suppléer aux caracteres particuliers d une Nation, par d'autres caracteres qui conviennent à ceux poutr quI l'on traduit. Pour moi, je crois avec plusieurs autres personnes, que j'ai consultées sur ce précepte, qu'une pareille Traduction ne pourroit passer, tout au plus, que pour une espèce de Comparaison. $^{13}$

Le texte étranger présente ici un « tour heureux » que la censure rendrait impossible dans le polysystème français. L'importance de la fidélité de la traduction d'un tel texte est soulignée par l'auteur avec autant de force que s'il s'agissait d'un texte plus canonique ou plus "sacré " que celui d'un journal anglais. La transposition des types ou des métaphores que certains prônent au nom de la fidélité aux types humains ou aux « caracteres particuliers d̀ une Nation ", n'est, pour l'auteur, que cette « espece de Comparaison $"$.

Soulignons également le rôle des « personnes consultées » : la répétition des formules et l'absence de précisions semblent de prime abord peu convaincantes, mais elles montrent l'importance accordée a l'opinion de spécialistes ou de simples lecteurs qui verraient dans la fidélité un respect des caractéristiques du texte de départ. L'auteur de cette préface, tout comme Dacier qui avoue qu'elle se reconnaît « tres inferieure à [son] Original; de maniere [qu'elle] a cru devoir soutenir [son] travail par des Remarques qui

13. "Préface ", le Free-Holder, ou l'Anglois jaloux de sa Liberté (Amsterdam, Uytwerf, 1727); cité par Graeber (1990), p. 81. 
fissent sentir les beautez [qu'elle n'avait] p0 exprimer ", l'auteur de la Préface du Free-Holder, donc, ne cherche pas non plus l'équivalence à tout prix et se demande si, pour faire mieux comprendre les « caracteres propres à une Nation 》, il ne vaudrait pas mieux « avoir recours à une Note, pour tâcher de les faire sentir à ceux pour qui l'on traduit ${ }^{14}$ ".

Ce point de vue semble partagé par Silhouette dans ses "Réflexions préliminaires du traducteur 》, en tête de l'Essai sur la critique et sur l'homme ${ }^{15}$, où il s'oppose à la traduction en vers du même texte par Du Resnel (1737) ${ }^{16}$. Malgré la " gêne perpétuelle " de l'assujettissement à une "imagination étrangère " et la "différence des langues", et s'il rejette les traductions trop littérales au nom de l'art qui doit éviter tout excès, Silhouette (1737) considère que l'" exactitude » et la " fidélité " constituent l'essence de la traduction qu'il compare à l'histoire :

14. Cité par Graeber (1990), p. 81.

15. Alexander Pope, Esssay on Criticism, 1711, Essay on Man, 17331734.

16. « Popes Werke lieferten den französischen Übersetzern [...] den heftigsten Anlaß zur Diskussion um Treue bzw. Freiheit gegentber dem Original. Wahrend die Versubersetzer in der Regel eine Wiedergabe des Sinnes für hinreichend halten, betonen die Prosatibersetzer Popes Bedeutung als Philosoph; da seine Gedanken bereits durch kieinste tbersetzerische Abweichungen entstellt würden, pladieren sie fur weitestgehende Treue. " « Les cuvres de Pope donnaient lieu chez les traducteurs français aux discussions les plus passionnées au sujet de la fidélité et de la liberté par rapport à l'original. Alors que les traducteurs en vers considéraient en général la transmission du sens comme suffisante, les traducteurs en prose soulignaient le rôle de Pope comme philosophe et, puisque le sens de ses idées serait détourné par les plus minimes déviations traductionnelles, ils se faisaient les avocats d'ume traduction la plus fidèle possible. ) (Graeber, 1990, p. 95, notre trad.) Morris (1961, p. 331) recense ainsi cinq traductions differentes de The Rape of the Lock avant 1750. 
L'une rapporte les pensées ainsi que l'autre rapporte les faits, \& il est aussi peu permis d'en altérer les circonstances que le fonds. Les changer, c'est faire injustice à son Héros ou à son Auteur, \& encore plus à ce dernier : car il n'est guères d'Auteurs, il n'en est peut-être point qui ne préférât d'être traduit fidélement, comme il n'est guères de traducteurs quj ne préférassent de traduire librement, c'est a dire, en se donnant la permission d'altérer l'original. L'éxactitude est un assujétissement pénible \& peu glorieux. (p. 5)

Le rôle du traducteur " assujetti » est pour Silhouette celui du peintre copiant l'original; il fait siens les propos de Tourreil pour qui, déjà au XVII ${ }^{\circ}$ siècle, « tout Copiste qui dérange seulement les traits, ou qui les façonne à sa mode, commet une infidélité. " Il trompe alors \& sous le nom de truchement; [il] ne tradui[t] point, [il] produi[t]. ${ }^{17}$ " L'assujettissement dont il est ici question rappelle la soumission associée au concept de fidélité à un maître dans les dictionnaires susmentionnés, le maître étant bien sûr ici le texte de départ.

Malgré les compromis inévitables, ce devoir, cet assujettissement à l'ceuvre à copier l'emporte donc sur les gouts de l'époque ou de la culture d'arrivée. Silhouette en arrive ainsi à la question de l'antériorité des idées ou du mode d'expression et oppose la première à ceux qui croient que l'on doit trouver en français ce que Pope aurait écrit s'il avait pensé dans cette langue ${ }^{18}$. Il en conclut qu'il faut rester fidèle aux idées de l'auteur et même en conserver la concision, l'obscurité, quitte à l'expliciter dans une préface. Le programme de Silhouette semble donc en opposition avec la poétique des belles infidèles puisqu'il entend préserver le caractère étranger, tant au niveau de la forme que de la thématique, d'un texte a la fois philosophique, anglais et quasi contemporain. Les deux dernières caractéristiques sont importantes,

17. Cite par Silhouette, p. 4.

18. « Il y a quelque chose d'antérieur aux expressions, ce sont les idées », cité par Graeber (1990), p. 99. 
puisqu'elles ne se retrouvaient pas dans les textes traduits et les écrits théoriques dont il a été question jusqu'à présent.

\section{La fidélité selon Desfontaines}

Chez Desfontaines, les expressions " fidèle 》, " fidélité 》 ou " fidèlement » reviennent constamment, tant dans ses articles que dans ses préfaces. Les articles des Observations et des Jugements lui permettent de commenter les traductions d'auteurs anciens ou (plus rarement) modernes, et de revenir sur le rôle de la fidélité. Il n'hésite pas non plus à critiquer ses propres traductions, en se les attribuant ou non, et en les comparant à d'autres.

Si l'expression " fidèle » et ses dérivés sont récurrents sous la plume du journaliste, il n'en demeure pas moins difficile d'extraire une définition. Il déclare que la fidélité " sera toujours la première qualité du Traducteur " et que sans elle " il n'en merite pas le nom " (Observations, lettre XLI, p. 248), ou qu'une traduction des Confessions de saint Augustin par Hennequin est " estimable " par la " pureté du texte \& la fidélité de la traduction 》 (Observations, lettre CCCCIII, p. 290), mais la seule manière de cerner le sens du concept semble être de retenir quelques groupements phraséologiques particulièrement récurrents chez Desfontaines :

fidéle, exacte, précise, tendre, élevée \& nerveuse (Jugements, vol. I, t. 1, p. 18)

de la fidélité \& de l'exactitude dans les traductions (Observations, lettre XDV, p. 431)

fidéle, exact \& même littéral (Ibid., lettre CCCCLXXVII, p. 270) La fidélité \& l'exactitude [...] exacte fidélité (Ibid, lettre CCCCIII, p. 331)

Au-delà des commentaires stylistiques (" tendre, élevée \& nerveuse "), c'est l'" exactitude » qui est le plus souvent associée à la fidélité, dans une relation quasi synonymique. Le contexte de ces cooccurrences indique cependant que l'association fidélité-exactitude constitue l'un des principes de la traduction, et 
non un absolu pour Desfontaines : la première cooccurrence citée a trait à la traduction idéale de Tibulle que Desfontaines attend encore; dans la seconde il précise que, même s'il est " très-ami » de la fidélité et de l'exactitude dans les traductions, il importe de se souvenir que le français exige justesse et précision, et que le traducteur des Anciens ne doit pas être trop attaché à la lettre. Dans le troisième exemple, il devient évident que l'exactitude " servile " ne constitue pas un mérite de la traduction en prose. Dans le dernier cas enfin (sa propre traduction de Virgile), il précise que l'exactitude a étế son objet, mais qu'il a gardé à l'esprit les caractéristiques des deux langues.

La première constatation semblerait donc être que Desfontaines voit dans la fidélité un absolu, un idéal qui ne peut qu'être subordonné à des contingences propres à l'activité traduisante : langue de départ, langue d'arrivée, public visé, type de texte, etc. Mais il est plus difficile de cerner sa poétique traductionnelle (si poétique il y a) en matière de fidélité. Il reproche ainsi à d'Ablancourt l'infidélité de ses traductions : « d'Ablancourt, dont le stile en général est excellent, n'est point un Traducteur fícéle; [...] il omet ce qu'il n'a point entendu, \& [...] il altére souvent ce qu'il a pu entendre. Voilà le grand défaut de ses traductions. " (Observations, lettre CCCCV, p. 86) Et pourtant Desfontaines ne cache pas qu'il n'a pas toujours pu être fidèle, même dans le cas des Anciens : le traducteur français devant toujours, pour lui,

se souvenir qu'il écrit dans une langue qui aime la justesse et la précision, \& que c'est défigurer \& avilir les Auteurs de l'antiquité, $\&$ ne pas sçavoir traduire, que d'être trop attaché à la Lettre, \& de ne pas quelquefois resserrer en peu de paroles ce qui est trop long dans l'original; de même le Traducteur doit quelquefois développer telle pensée, pour la faire entendre \& gouter dans sa langue, mais sans paraphrase. (Observations, lettre XDVIII, p. 44)

Dans la traduction des Anciens et, a fortiori, des Modernes, la fidélité paraît donc être pour Desfontaines un principe traductionnel facultatif. 
Le peu d'importance accordée de facto à la fidélité atteint un de ses sommets dans la traduction des Voyages de Gulliver et il n'est pas inutile de citer quelques extraits révélateurs de la poétique de Desfontaines' ${ }^{19}$ : il avouera avoir traduit l'Achille dans l'Isle de Sciros " le plus fidelement qu'il [lui avait] été possible ${ }^{20}$ ", mais la fidélité n'est déjà plus avec Gulliver synonyme de soumission; elle est plutôt elle-même soumise aux impératifs du goût français, au bon goût et à la vraisemblance exigés par les critiques.

Desfontaines, connu pour son caractère emporté et une fatuité sans pareille, n'hésite pas à le proclamer à Swift lui-même dans une lettre : " J'ai voulu donner aux François un livre, qui fut (sic) à leur usage : voilà ce qui m'a rendu traducteur libre et peu fidèle (Swfit, 1883, p. 119). À propos de Swift et de l'historien Echard, il écrit ensuite dans les Observations : « La réputation de ces Auteurs n'étoit pas assez grande, pour m'asservir à leurs pensées. [...] Voilà, ce me semble, l'espèce de Livres qui dispensent de la fidélité ceux qui entreprennent de les traduire. 》 (Lettre XLI, pp. 246-247) Il est donc clair que, dans le cas d'auteurs (quasi) contemporains, le traducteur ne se sent pas « asservi » à la fidélité.

En fait, dans la traduction des ouvrages contemporains, la fidélité est associée continuellement par Desfontaines au mot à mot, à une littéralité inacceptable. Sans l'avoir lue (il l'avoue lui-même ${ }^{21}$ ), il revient ainsi à plusieurs reprises sur la traduction des Voyages de Gulliver publiée à La Haye la même année que la

19. Pour une description des « fautes » et « infidélités » du traducteur, cf. Morris (1961), chap. V.

20. " On conçoit cependant que je n'ai pû être toujours Traducteur litteral, à cause de la différence extrême des deux Langues. Cependant je ne me suis jamais éloigné du sens de l'Original; j'ai omis fort peu de chose, \& je n'ai rien mis dans ma Traduction, qui ne soit réellement ou equivalemment dans le Texte. " Desfontaines (1737) : " Préface du traducteur 》, p. jv.

21. Desfontaines (1727), « Préface du traducteur », p. xii. 
sienne et connue pour sa littéralité; par exemple quand Silhouette lui reproche d'avoir gommé des passages que Desfontaines considérait comme trop grossiers ou trop bas :

On peut voir toutes ces gentillesses dans la traduction fidéle \& littérale, imprimée en Hollande, \& que personne n'a pâ lire à Paris. Celle de M. L'Abbé D. F. a été réimprimée plusieurs fois, même en Hollande, où au contraire la Traduction littérale n'a pa trouver d'acheteurs. (Observations, lettre CLXXXVIII, p. 172)

La traduction implique pour Desfontaines fidélité, mais en fait, pour lui, les ouvrages contemporains ne méritant pas d'être " traduits ", il n'est donc pas tenu de demeurer fidèle.

Avec la traduction de Joseph Andrews ${ }^{23}$, le rapport de Desfontaines à l'étranger serait en train de changer, selon Graeber : alors qu'il s'était vanté des libertés prises en traduisant les Voyages de Gulliver, Desfontaines tendrait maintenant à la «fidélité linguistique " (philologische Treue) (Graeber, 1990, p. 110) ${ }^{24} . \mathrm{Si}$ l'on pose la fidélité sur un axe allant de la fidélité au texte de départ à la fidélité au polysystème d'arrivée, la place d'un Desfontaines se situe bien sûr du côté du second pôle; tout changement dans sa poétique paraîtra alors révolutionnaire. Dans Joseph Andrews, Desfontaines ne se vante plus de ses infidélités, mais il les justifie encore par la différence de goat entre les nations; son plus grand mérite semble cependant être de longuement présenter la culture anglaise dans ses instances liminaires.

22. Cf. aussi la préface du « traducteur " de la Boucle de cheveux enlevée (Desfontaines, 1728), p. xi : « [L'ouvrage de Swift] avoit besoin qu'on y mit la main, sur tout depuis qu'on a vu à Paris ces mêmes Voyages de l'édition d'Hollande, traduits fidélement, \& mot à mot. "

23. Desfontaines, H. Fielding (1743).

24. Graeber (1990), p. 110. 
Le roman. de Fielding est pourtant traduit seize ans après celui de Swift; on peut donc se demander si l'anglomanie en plein essor aura modifié les idées de Desfontaines qui a de plus traduit de nombreux autres ouvrages entre temps. Les conditions de travail (le Gulliver avait été traduit très rapidement pour que cette traduction soit la première) et la connaissance de l'anglais de Desfontaines ont dû également influer sur sa conception de la fidélitée ${ }^{2 s}$.

Desfontaines est loin d'être le seul traducteur de l'époque à se vouloir fidèle en théorie mais à reconnaître que la fidélité doit se soumettre au bon goût du polysystème d'arrivée : ce point de vue est partagé par La Place, Fréron et Yart, et la soumission de la fidélité semble devenir le trait caractéristique de la poétique traductionnelle de ce second tiers du XVII' siècle, dans le cas du moins de la littérature anglaise. La Place, premier traducteur français de Shakespeare, cherche le juste milieu entre la fidélité à l'auteur et les usages français; la fidélité devient encore une fois un handicap qui peut nuire à l'auteur :

Si je veux sauver certains traits trop révoltans pour nous, les Anglois diront que j'aurois forcé, détourné, ou rendu foiblement le sens de l'Auteur. Si je les rens fidélement, l'Auteur y perdra parmi nous; \& les deux Nations me rendront également responsable de ce qui ne flattera pas le goat de l'une, \& l'amour-propre de l'autre. ${ }^{26}$

25. Il est difficile de savoir jusqu'où allaient les connajssances de Desfontaines à l'époque de la traduction des Voyages de Gulliver; les critiques, tant contemporains que modernes, ont plus ou moins sévèrement remis en question sa connaissance de l'anglais (Moreri, dans son Dictionnaire [1759], lui refuse toute compétence, tandis que Morris fait le décompte de ses " fautes" et " infidélités ". On peut cependant supposer que cette connaissance, à partir d'un niveau $x$, a continué à se développer.

26. "Discours sur le théatre anglois ", le Thédtre anglois (Londres, Nourse, \& Paris, 1746-1749), cité par Graeber (1990), p. 114. 
Fréron met quant à lui la fidélité en dessous de la beauté d'une traduction qui enrichit l'original en lui attribuant les critères esthétiques français: "Rien n'est plus aisé qu'une fidélité scrupuleuse; rien ne l'est moins que le bel art d'embellir et de perfectionner ${ }^{27}$ \%. Yart enfin, qui tente de concilier fidélité, élégance et littéralité :

[...] j'ai réuni, autant qu'il m'a été possible, la fidélité \& l'élégance : mais quand $\mathrm{j}$ 'ai trouvé des expressions, des métaphores, des tours purement Anglois, qui pourroient choquer la delicatesse de notre goat, je les ai adoucis dans le texte, \& en ai donné la traduction littérale dans les notes. ${ }^{2 \pi}$

Mentionnons enfin un cas plus tardif qui souligne bien l'importance accordée à la notion de fídélité par les traducteurs tout au cours du XVIII' siècle. Il s'agit encore une fois de la traduction d'une cuvre moderne: La Gerusalemme liberata du Tasse par Panckoucke (1785). Le débat sur la traduction des textes en vers ou en prose se poursuit ainsi jusqu'en 1785 alors que Panckoucke constate que les deux traductions existantes de la Jérusalem libérée different profondément. Face à l'éternelle question des différences entre les traductions d'un même texte, il se propose un but complètement différent :

j'aurais voulu faire une traduction littérale et fidèle qui pat être imprimée à côté du texte, qui ne fut destinée qu'à cet usage, qui rendít le génie du Tasse, et qui servit en même temps à faciliter la lecture et l'intelligence de ce grand Potte ${ }^{29}$.

27. Fréron, Année littéraire, 1756, VI, p. 243; cité par Morris (1961), p. 271.

28. Yart, " Préface ", Idée de la Poésie Angloise, p. xv (Paris, Briasson, 1749-1756); cité par Graeber (1990), p. 122.

29. Panckoucke, " Préface ", la Jérusalem libérée, 1785; cité par StPierre (1990), p. 119. 
Cette position pédagogique qui conçoit la traduction comme un médium permettant de mieux lire l'original s'applique cependant à un texte italien et relativement ancien, qui fait presque partie, dejjà pour cette époque, des classiques.

\section{Conclusion}

Il serait présomptueux de vouloir ici établir une Histoire de la fidélité en traduction au XVII' siècle, d'abord à cause du grand nombre de textes publiés alors sur ce sujet, que ce soit sous la forme de préfaces, d'articles ou de pamphlets. Mais surtout parce que, même si ce travail préalable semble montrer qu'une conception de la fidélité l'emporte à un moment ou à un autre (soumission à l'étranger ou à la culture d'arrivée), il n'en demeure pas moins que toutes les conceptions de la fidélité, concept particulièrement vaste $^{30}$, co-habitent et évoluent au cours de la période qui nous intéresse, comme dans le cas de n'importe quelle autre période. Il est plus intéressant par contre de noter que l'expression " fidélité " demeure profondément polysémique et qu'elle peut avoir, selon les points de vue, des sens diamétralement opposés.

On constate cependant un glissement général dans la conception de la fidélité : la fidélité au texte étranger ou à la langue d'arrivée, considérée auparavant comme un absolu par Méziriac ou Godeau, devient . une caractéristique presque accessoire chez Desfontaines. Il importe pourtant de noter que le changement ne se fait pas pour n'importe quel type de texte : le texte classique gréco-latin (ou même celui de la Renaissance italienne) continue de faire l'objet d'une traduction qui se veut profondément " fidèle ", qu'il s'agisse de respecter le contenu ou la langue d'Homère

30. «La notion de 'fidélité', par exemple, peut recevoir des sens assez différents selon les contextes et selon les traducteurs. $\mathrm{Si}$, pour certains, traduire fidèlement signifie rendre l'expression même du texte original, pour d'autres, une traduction fidele est celle qui se libère de l'expression pour serrer de plus près l'intention de l'auteur, le message du texte. » (St-Pierre, 1990, p. 124, n. 2) 
(comme le veut Dacier) ou ce que l'on pourrait appeler la " dignité " de l'auteur, en gommant ses " erreurs " pour le mettre au goût du jour et lui être ainsi encore plus fidèle (Houdar de La Motte). Seul le texte étranger contemporain (et en particulier le roman anglais, dans le cas qui nous intéresse) n'exige plus de son traducteur une absolue fidélité.

La traduction des auteurs contemporains anglais se présente done sous un tout autre jour. $A$ la poésie antique s'oppose ici la prose anglaise, romanesque ou scientifique : Desfontaines en est l'un des premiers traducteurs et, ce qu'il pose comme programme de traduction, est une véritable adaptation. Le texte anglais n'impose pas le respect, la gloire des auteurs n'est pas assez grande et, enfin, certains comportements et certains récits sont littéralement inacceptables pour le traducteur et pour le public français.

On en arrive donc à la paradoxale conclusion que la notion de fidélité, même si elle revient continuellement sous la plume de Desfontaines, ne s'applique pas dans le cas des traductions de la littérature anglaise. C'est qu'en fait la traduction pour Desfontaines ne semble concerner que la littérature ancienne, tandis que la littérature moderne fait l'objet d'une adaptation. Tandis que la fidélitê religieuse (fides) de la traduction-version des textes religieux et la soumission (traduction) aux textes anciens relèvent encore ici d'un souci d'" exacte fidélité " à l'esprit et à la lettre de l'original, la traduction des ceuvres contemporaines, elle, relève de la fidélité à la vérité (du souci de la vérité?) par l'adaptation. La lecture critique des traduction de Desfontaines devra donc se faire à partir du " projet de traduction " (Berman, 1995, p. 76) de celui-ci, et non à partir de nos conceptions modernes de la fidélité.

Université McGill 


\section{Références}

Académie française (1718). Nouveau Dictionnaire de l'Académie françoise. Genève, Slatkine Reprints, 1994.

BALlARD, Michel (1992). De Cicéron à Benjamin. Traducteurs, traduction, réflexions. Lille, Presses universitaires.

BEAUZÉE, Nicolas (1751-1765). "Traduction, version ", Encyclopédie ou Dictionnaire raisonné des sciences, des arts et des métiers. Paris \& Neuchâtel, S. Faulche \& Cie.

BERMAN, Antoine (1984). L'Épreive de l'étranger. Culture et traduction dans l'Allemagne romantique. Paris, Gallimard.

Gallimard.

(1995). Pour une critique des traductions. John Donne. Paris,

BURY, Emmanuel (1991). " Bien écrire ou bien traduire. Pierre-Daniel Huet théoricien de la traduction ", Littératures classiques. « La traduction au XVII" siècle " 13, Paris, Klincksieck, pp. 251-260.

CARY, Edmond (1963). " Madame Dacier, Houdar de La Motte et les 'Belles Infidèles' ", Les Grands traducteurs français. Étienne Dolet, Amyot, $\mathbf{M}^{\mathrm{m} \epsilon}$ Dacier, Houdar de La Motte et les traducteurs d'Homère, Galland et les traducteurs des Mille et une nuits, Gérard de Nerval, Valéry Larbaud. Genève, Georg, pp. 29-59.

CORNEILLE, Thomas (1694). Dictionnaire des arts et des sciences. Genève, Slatkine Reprints, 1968.

DACIER, Anne (1735 [La Haye]). Des causes de la corruption du goût. Genève, Slatkine, 1970.

(1766). Introduction à l'lliade. Leide, Wetstein \& fils [citée par Horguelin, pp. 113-114]. 
DESFONTAINES, Abbé (1727) Swift -. Les Voyages de Gulliver, traduit de l'anglois de Swift. Paris, Guérin.

(1728) Pope -. La Boucle de cheverx enlevée, Poème héroïcomique de M. Pope. Traduit de l'Anglois, par M. L. D. F. Paris, Le Breton père.

(1735-1743 [Paris]). Observations sur les écrits modernes. Genève, Slatkine, 1967. Chaubert.

(1737) Métastase -. Achille dans l'Isle de Sciros. Paris, (1743) Fielding -. Les Avantures de Joseph Andrews, et de son ami Abraham Adams, Londres, A. Millar.

(1744-1746 [Avignon]). Jugements sur quelques ouvrages nouveanx. Genève, Slatkine, 1967.

FOUCAULT, Michel (1969). L'Archéologie du savoir. Paris, Gallimard.

(1991). "Politics and the Study of Discourse ", The Foucault Effect. Studies in Governmentality. G. Burchell dir. et al. Chicago, The University of Chicago Press, pp. 53-72.

FURETIÈRE, Antoine (1690). Dictionnaire universel, contenant généralement tous les mots françois tant vietax que modernes... Rotterdam \& La Haye, Arnout \& Reinier Leers.

GRAEBER, Wilhelm et Geneviève Roche (1988). Englische Literatur des 17. und 18. Jahrhunderts in französischer Obersetzung und deutscher Weitubersetzung... Tubingen, N. Niemeyer.

dir. (1990). Französische Übersetzervorreden des 18. Jahrhunderts. Francfort/Main, P. Lang. 
(1992). " Wandeln in der kulturellen Fremderfahrung 》, Die literarische Übersetzung als Medium der Fremderfahrung. F. Lonker, dir., Berlin, E. Schmidt, pp. 71-86.

GUELLOUZ, Suzanne (1991). « Du bon usage des textes liminaires. Le cas d'Amelot de la Houssaye ", Littératures classiques. "La traduction au XVII siècle ", 13, pp. 261-275.

HORGUELIN, Paul A., dir. (1981). Anthologie de la manière de traduire. Domaine français. Montréal, Linguatech.

MATORÉ, Georges (1968). Histoire des dictionnaires français. Paris, Larousse.

MORRIS, Thelma (1961) : " L'Abbé Desfontaines et son rôle dans la littérature de son temps ", Studies on Voltaire and the Eighteenth Century, 19.

PRÉVOST, Abbé (1750). Manuel lexique, ou Dictionnaire portatif... [nouv. éd. consid. revue et augm., Paris, Didot, 1755].

RÉAU, Louis (1938 et 1971). L'Europe française au temps des Lumières. Paris, Albin Michel.

RICHELET, Pierre (1680). Dictionnaire français contenant les mots et les matières..., Genève, Widerhold.

SILHOUETTE, Étienne de (1737). « Réflexions préliminaires du traducteur sur le goût des traductions », Pope - Essai sur la critique et sur l'homme. Nouv. éd., Londres, G. Smith.

ST-PIERRE, Paul (1990). "La Traduction. histoire et théorie ", Meta, XXXV (1), pp. 119-125.

(1993). "Translation as a Discourse of History ", TTR, VI (1), pp. 61-82. 
SWIFT, Jonathan (1727 [1883]). " Lettre du 4 juillet 1727 ", The Works of Jonathan Swift, W. Scott, éd., Londres, Bickers, XVIII, p. 119.

VOLTAIRE (1974). Commentaires sur Corneille. Édition critique de David Williams, III. The Voltaire Foundation, Thorpe Mandeville House, Banbury, Oxfordshire.

WEIL, Françoise (1986). « L'Abbé Desfontaines et quelques autres traducteurs de l'anglais ", in Jean Varloot, dir., $D u$ baroque aux Lumières. Rougerie, pp. 114-116.

ZUBER, Roger (1968). Les "Belles infidèles " et la formation du goût classique. Perrot d'Ablancourt et Guez de Balzac. Paris, A. Colin.

RÉSUMÉ : Soumission et assujettissement : la fidélité chez les traducteurs et « théoriciens » de la traduction française dans la première moitié du XVIII' siècle - Cet article porte sur le concept de fidélité en traduction en France au XVIII ${ }^{\circ}$ siècle. À partir des définitions des dictionnaires de la fin du XVII et du début du XVIII' siècle, on cherche à définir la visée de la fidélité à l'époque : fidélité au texte de départ ou à la culture d'arrivée? Les dictionnaires définissent d'abord le terme par rapport à l'exactitude, à la foi et à la soumission. Les débats sur la fidélité dans la traduction des Anciens entre Houdar de La Motte et Anne Dacier au début du $\mathrm{XVIII}^{\circ}$ siècle se réfletent dans la traduction de la littérature anglaise. L'assujettissement $d u$ traducteur devient avec Silhouette celui du copiste. La fidélité est associée par Desfontaines à l'exactitude, mais en tant qu'idéal elle ne peut être selon lui que soumise aux contingences de la traduction et de la langue française. La fidélité devient alors synonyme de littéralité et Desfontaines, comme d'autres traducteurs, choisit plutôt d'être fidèle au gout français. L'exigence même de fidélité se module selon le type de texte à traduire : la Bible exigera une version littérale, les Anciens une 
imitation cherchant même à rendre justice à l'auteur en l' " améliorant », et les auteurs contemporains dont la " gloire » n'est pas assez importante, n'auront droit qu'à une adaptation libre.

ABSTRACT: "Soumission" and "Assujettissement": Fidelity for Translators and "Theoreticians" of French Translations in the First Half of the Eighteenth Century - This article analyzes the concept of fidelity in translation in France during the first half of the eighteenth century. On the basis of definitions provided by dictionaries from the late seventeenth century and the beginning of the eighteenth century, the article attempts to define the scope of fidelity at that time: fidelity to the source text, or to the target culture? The dictionaries first explain the concept in terms of exactness, faith or submission. The quarrel between Houdar de La Motte and Anne Dacier regarding faithfulness in the translation of the Ancients is later reflected in the translation of English literature. With Silhouette, the translator's submission becomes that of a copyist. Desfontaines associates the idea of fidelity with that of "exactness," but only as an ideal which for him is strictly subjected to the contingencies of translation and of the French language. Thus, fidelity becomes synonymous with unacceptable literal translation; Desfontaines, like other translators, chooses to be faithful to French taste. The requirements of fidelity itself change according to the nature of the text to be translated: the Bible demands a literal version, the Ancients must be imitated and the translator should do justice to these authors by "improving" their work. Finally, contemporary authors whose "glory" is not recognized, will only be given a free adaptation.

Benoit Léger : Département de langue et littérature françaises, Université McGill, 3460, rue McTavish, Montréal, Québec H3A 1X9, CANADA.

Courriélec : bpn6@musicb.mcgill.ca 